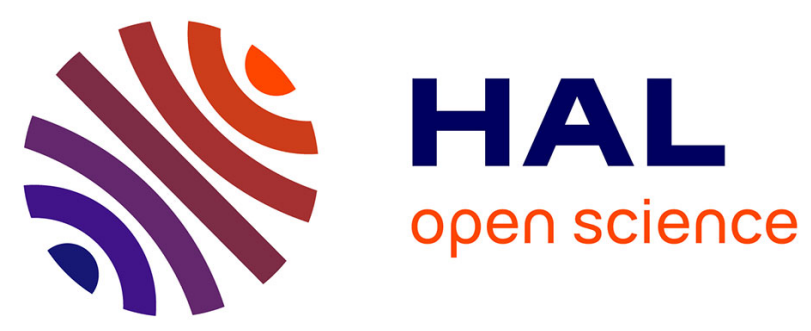

\title{
Combining micro- Meso- And macro-scopic numerical methods for multiscale radiative transfer modeling of SiC-based foams up to very high temperatures
}

Mohd Afeef Badri, Arnaud Biallais, Gilberto Domingues, Y. Favennec, Giorgia Fugallo, Aubin Mekeze Monthe, Benoit Rousseau

\section{To cite this version:}

Mohd Afeef Badri, Arnaud Biallais, Gilberto Domingues, Y. Favennec, Giorgia Fugallo, et al.. Combining micro- Meso- And macro-scopic numerical methods for multiscale radiative transfer modeling of SiC-based foams up to very high temperatures. 16th International Heat Transfer Conference, IHTC 2018, Aug 2018, Beijing, China. 10.1615/IHTC16.pma.024216 . hal-02376182

\section{HAL Id: hal-02376182 \\ https://hal.science/hal-02376182}

Submitted on 10 Dec 2019

HAL is a multi-disciplinary open access archive for the deposit and dissemination of scientific research documents, whether they are published or not. The documents may come from teaching and research institutions in France or abroad, or from public or private research centers.
L'archive ouverte pluridisciplinaire HAL, est destinée au dépôt et à la diffusion de documents scientifiques de niveau recherche, publiés ou non, émanant des établissements d'enseignement et de recherche français ou étrangers, des laboratoires publics ou privés. 
See discussions, stats, and author profiles for this publication at: https://www.researchgate.net/publication/325673033

COMBINING MICRO- MESO- AND MACRO-SCOPIC NUMERICAL METHODS FOR MULTISCALE RADIATIVE TRANSFER MODELING OF SiC-BASED FOAMS UP TO VERY HIGH TEMPERATURES

Conference Paper · August 2018

DOI: 10.1615/IHTC16.pma.024216

CITATION

1

7 authors, including:

Benoit Rousseau

French National Centre for Scientific Research

103 PUBLICATIONS 682 CITATIONS

SEE PROFILE

Yann Favennec

Polytech Nantes

69 PUBLICATIONS 517 CITATIONS

SEE PROFILE

Some of the authors of this publication are also working on these related projects:

Numerical Solution of Radiative Transfer in Participating Media View project

Spectral radiative properties of dispersed materials View project
READS

260

Gilberto Domingues

University of Nantes

21 PUBLICATIONS 366 CITATIONS

SEE PROFILE

Mohd Afeef Badri

Atomic Energy and Alternative Energies Commission

18 PUblications 35 Citations

SEE PROFILE 
IHTC16-24216

\title{
COMBINING MICRO- MESO- AND MACRO-SCOPIC NUMERICAL METHODS FOR MULTISCALE RADIATIVE TRANSFER MODELING OF SIC-BASED FOAMS UP TO VERY HIGH TEMPERATURES
}

\author{
M. A. Badri ${ }^{1,2}$, A. Biallais ${ }^{3}$, G. Domingues², Y. Favennec², G. Fugallo², A. Mekeze Monthe², B. Rousseau ${ }^{2, *}$ \\ ${ }^{1}$ IRT Jules Verne, Chemin du Chaffault, 44340 Bouguenais, France \\ ${ }^{2}$ CNRS, LTeN, UMR 6607, Université de Nantes, Rue Christian Pauc, 44306 Nantes Cedex 3, France \\ ${ }^{3}$ Ouest Valorisation, 30 bd Vincent Gâche, CS 70211, 44201, Nantes Cedex, France
}

\begin{abstract}
A general multiscale numerical approach is proposed to finely reproduce, up to $1300 \mathrm{~K}$, the radiative heat transfers within alpha silicon carbide-based open-cell foams, with tuned chemical and textural features. The complete modeling is based on a thorough analysis performed at micro-, meso-, and macro-scopic scales. To gauge, at the microscopic scale, the influence of the electron-phonon coupling on the intrinsic optical properties of silicon carbide, a molecular dynamic method involving a modified Tersoff potential is used. Then, at the mesoscopic scale, with optical indices provided by our molecular dynamic simulations, a collision-based Monte Carlo ray tracing method is used for retrieving the homogenized radiative properties of the selected foams. Finally, at the macroscopic scale, for a set of foams (porosity $\sim 0.4-0.9$ ), we used our micro-meso constructed radiative properties for calculating the propagation of radiation by solving the radiative transfer equation using the vectorial finite element method. In addition, a fictitious foam constructed using heavily chemical doping at microscopic level, is put forward to change the volumetric propagation of the thermal radiation.
\end{abstract}

KEY WORDS: Radiation, Porous Media, Numerical simulation, Electron-phonon coupling, Molecular Dynamics

\section{INTRODUCTION}

Silicon carbide ( $\mathrm{SiC}$ )-based open-cell foams are, today, considered as attractive porous media for developing hightemperature conversion energy systems (up to $1400 \mathrm{~K}$ ). For example, they may be used in, porous gas burners [1], volumetric solar receivers [2] or, heat exchangers [3] among others. The growing interest for this family of material is firmly linked to the outstanding high-temperature properties of SiC, when it is considered as a dense material. Further, SiC-based dense ceramics exhibit high thermal conductivities, good corrosion resistance [4], low thermal expansion coefficients, and high strengths [5] which confers them a good thermal shock resistance, even in aggressive atmosphere.

However, the knowledge of the thermal dependence of the main physical properties of SiC-based dense materials is insufficient to gauge accurately the effective physical properties of SiC-based open-cell foams. This is the case for the effective thermal conductivity, especially when thermal radiation dominates heat transport for temperatures higher than $1000 \mathrm{~K}$ [6]. On one hand, experimental characterizations are carried out with difficulty above $1000 \mathrm{~K}$ [7] and, on the other hand, mathematical models allowing the quantification of the exact contribution of the radiative conductivity can lead to discordance. This lack of understanding leads, either to approximate design of the conversion systems or to overconsumption of energy for maintaining the operating temperature. This questioning is directly related to the role played by the complex multiscale organization of these porous materials on their radiative heat transfers.

*Corresponding Author: benoit.rousseau@univ-nantes.fr 
It is important to notify that $\mathrm{SiC}$ is a semiconductor material for which the intrinsic optical properties in the infrared spectral range is mainly governed by the interaction between the electronic carriers induced by the chemical doping and its atomic lattice [8]. As an illustration of this effect, a strut with a thickness of $200 \mu \mathrm{m}$ can be opaque in the infrared range when the carriers concentration is important and rather transparent when this concentration decreases drastically [9]. At the mesoscopic scale, the layout of struts, walls and cells that form the foam, influences the transport of the radiative energy by promoting either scattering or absorbing phenomena with some weights that can be radically different if the volume of the cellular medium contains or not the Representative Elementary Volume $(R E V)$ for identifying the homogenized radiative properties [10]. Lastly, at the macroscopic scale, the determination of the radiative propagation can suffer from inexactness in reason of the several assumptions required to simplify the radiative transfer equation that is, from a mathematical viewpoint, an integrodifferential equation for which analytical solutions are uncommon [11].

To cope with this multiscale issue, a global numerical approach is proposed in this work to take into account the main factors that govern, scale by scale, the radiative heat transfers trough virtual SiC-based open-cell foams with tuned properties. To generate the virtual foams, a homemade software, called genMat [12] is used. This approach allows predicting radiative heat transfers within a wide range of samples with fine tailored material parameters that is difficult to achieve with real elaboration processes. At the microscopic scale, molecular dynamics simulations are proposed with the aim to modify the complex refractive indices of SiC single crystals [13] only with the knowledge of the carrier concentration, after a careful analysis of the particular optical signature due to the electron-phonon coupling. Then, at the mesoscopic scale, a collision-based Monte Carlo ray tracing method is applied on the set of foams in order to identify the homogenized radiative properties [12]. Finally at the macroscopic scale, a fast three-dimensional method based on the vectorial finite elements is employed to compute the radiative flux crossing the whole set of porous samples [14]. This work will help us to propose solutions for modifying the volumic propagation of thermal radiation within porous $\mathrm{SiC}$ foams.

\section{NUMERICAL METHODOLOGIES}

\subsection{Molecular dynamics for the computation of the complex dielectric function at microscale}

In this part, in the framework of the molecular dynamics (MD) technique, the Lorentz-Drude (LD) model is used for evaluating the complex dielectric function $\tilde{\epsilon}_{\alpha \beta, v}$, of synthetic SiC-based crystalline domains with variable carrier concentration $n_{c}, \alpha$ and $\beta$ denoting the directions and $v$ the frequency. The validity of the LD model $\tilde{\epsilon}_{L D, v}$, for describing the optical responses of semiconducting materials is already well established as described in Ref. [8]. Then, by using MD simulations it is firstly possible to extract the optical signatures of the lattice vibrations (phonons), such as their transverse optical (TO) frequency $v_{T O}$, longitudinal optical ( $L O$ ) frequency $v_{L O}$, and the associated phonon damping coefficient $\Gamma$. Secondly, it is also possible to quantify the optical activity of the collective oscillation of carriers or plasmon depicted by the plasma frequency $v_{P}$, and its plasma damping $\gamma_{P}$. Let

us recall also that $v_{P} \sim \sqrt{n_{c}}[8,10]$. Before explaining the MD scheme, let us introduce some major assumptions for it combined used with the LD model.

Firstly, the computation will be only performed with the cubic crystallographic structure of SiC (Zinc Blende, ZB). Even if high-temperature samples exhibit an hexagonal structure, the resulting anisotropy slightly affects the components of $\tilde{\epsilon}_{\alpha \beta, v}$ in the far infrared rang [15], so that the numerical results obtained through the ZB structure will still be representative of the reality. Secondly, for modifying the carrier concentration, nitrogen atoms will be used as substitute for the carbon atoms of the cubic lattice as it can be experimentally performed, for example, with chemical vapor deposition process for synthetizing n-type doped $\mathrm{SiC}$ [16]. $n_{c}$, is therefore straightforwardly given by $n_{c}=N_{N} / V$ where $N_{N}$ is the number of nitrogen atoms and $V$ the volume of the simulation cell. The incorporation of carriers leads therefore to the apparition within the SiC's structure of a plasmon that can couple with the $L O$ mode of the SiC's phonon [8]. The resulting mode, known as $L O P C$ for Longitudinal Optical phonon Plasmon Coupled, will be treated in the next part as a phonon-like type particle. Thirdly, the simulations will be 
IHTC16-24216

carried out only at $T=300 \mathrm{~K}$ since higher temperatures do not modify $\tilde{\epsilon}_{\alpha \beta, v}$, as it can be shown for $n_{c}=10^{19} \mathrm{~cm}^{-}$ ${ }^{3}$ by infrared emittance spectroscopy [17].

Once these basis are settled, the MD technique consists in applying the Newton's second law for computing all positions, $r_{i}$, and velocities, $\dot{r}_{i}$, for each atoms $i$ as a function of the time $t$. The interatomic potential chosen in this work is a modified version of the semi-empirical Tersoff many-body potential [18]. Briefly the Tersoff's potential depends on a repulsive pair potential and of attractive pair potential, multiplied by a cut-off function that confines the overall potential in a continuous and derivable way. This potential, through adjustable parameters, allows taking into account several kinds of atoms at it is the case for doped $\mathrm{SiC}$ with $\mathrm{Si}, \mathrm{C}$ and $\mathrm{N}$. However, this potential in its pure form tends to overestimate the spectral position of the SiC's phonon. By adding a torsion liketerm to the original potential of Tersoff, Halac et al.[19] gave a more accurate description of the phonon signature. Once the latter modified potential of Tersoff is selected, $\tilde{\epsilon}_{\alpha \beta}(v)$ is defined as follows:

$$
\tilde{\epsilon}_{\alpha \beta, v}=1-\frac{1}{V k_{b} T \epsilon_{V}} \int_{0}^{\infty} \frac{d}{d t}\left(\left\langle M_{\alpha}(0) \cdot M_{\beta}(t)\right\rangle_{0}\right) e^{-i v t} d t
$$

where, $M_{\alpha}$ is the dipolar moment in the direction $\alpha$, given by the expression $M_{\alpha}=\sum_{i=1, N} q_{i} r_{i \alpha} \cdot q_{i}$ is the effective charge of the $i$ atom, function of $v_{L O P C}$ [20]. $\epsilon_{V}$ is the vacuum dielectric function, $V$ the volume of the simulating box, $k_{b}$ the Boltzmann constant, $T$ the temperature of the atomic system and $\left\langle M_{\alpha}(0) . M_{\beta}(t)\right\rangle_{0}$ the autocorrelation function of the dipolar moment. Since MD can only give the dipolar moment of phonon-like type particles, only $v_{T O}, v_{L O P C}$ and $\Gamma$ will be identified from the simulation of the autocorrelation function with an analytical expression deriving from a damped Lorentz dielectric function model [13]. Finally, for a given value of $n_{c}$, $v_{P}$ is immediately computed and since $\mathfrak{R}\left(\tilde{\epsilon}_{L D, v_{L O P C}}\right)=0, \gamma_{P}$ is therefore easily deduced. This procedure makes that for a given value of $n_{c}, v_{T O}, v_{L O P C}, \Gamma, v_{P}$ and $\gamma_{P}$ are perfectly known that is advantageous for quantifying $\tilde{\epsilon}_{L D, v}$. The knowledge of $\tilde{\epsilon}_{L D, v}$ can be used for calculating the complex refractive index $\tilde{n}_{S i C, v}$, for SiC-like crystalline domains, since $\tilde{n}_{S i C, v}=\sqrt{\tilde{\epsilon}_{L D, v}}$.

\subsection{Collision-based Monte Carlo Ray Tracing method for computing radiative properties at mesoscale}

In the following, $\tilde{n}_{S i c, v}$, will be affected to the solid continuous part of virtual 3D open-cell foams. The dimensions of the major constituents (cells, struts) allow the validation of the geometrical optic approximation for the treatment of light-matter interaction at the mesoscopic scale. The methodology used here for governing the local transport of rays has been deeply discussed in [21]. In particular, only specular reflection will be considered when rays interact with struts. Furthermore, when rays penetrate within the struts they are considered as absorbed since the solid part that is probed is optically thick. A particular attention is paid to elaborate foams with a $R E V$ that permits to identify with a high confidence [10] their homogenized radiative properties $\beta_{v}, \kappa_{v}, \sigma_{s, v}$, and $\phi_{v}\left(u, u^{\prime}\right)$, namely their (spectral) extinction, absorption, scattering coefficients and spectral scattering phase function for the direction of incidence $u$ and scattering $u^{\prime}$. This framework allows us to use a collision-based Monte Carlo Ray Tracing method to retrieve the radiative properties. Briefly, a huge number of rays $N_{R}$, are emitted from points $M$ that are uniformly distributed in the fluid phase surrounding the solid parts of a given foam. The interaction of a ray at a point $I$ with the solid part determines if it can be extinct, absorbed or scattered. In particular, for the reproduction of an absorption event, a random number $\eta$ uniformly distributed, is selected and compared to the local reflectivity, $\rho_{\text {SiC, } v}=f\left(\tilde{n}_{S i C, v}, \tilde{n}_{\text {Fluid }, v}, \mu\right)$ given by the Fresnel law, where $\mu$ is the cosine of the angle of reflection defined between the opposite normal to a triangular facet and the direction of incidence $u$. If $\eta>\rho_{\text {SiC, } v}$ the ray is considered as absorbed and otherwise scattered. The direction $u^{\prime}$, of each reflected ray is stored and is used to determine $\phi_{v}\left(u, u^{\prime}\right)$. For each extinction events, the path-length to collision $s_{e, v}=\left|M I_{v}\right|$, is computed and stored (for an absorption event, the same thing is done with $s_{a, v}$ ). Once all rays have been launched, the spectral 
cumulative chord length distribution function, $G_{e, v}(s)$, and the absorption cumulative probability, $P_{a, v}(s)$, are defined as follows:

$$
G_{e, v}(s)=\int_{0}^{s} \frac{1}{N_{R}} \sum_{j=1}^{N_{R}} \delta\left(s^{\prime}-s_{e j, v}^{\prime}\right) d s^{\prime} \text { and } P_{a, v}(s)=\int_{0}^{s} \frac{1}{N_{R}} \sum_{j=1}^{N_{R}} \delta\left(s^{\prime}-s_{a j, v}^{\prime}\right) d s^{\prime}
$$

For Beerian SiC open-cell foams, $\beta_{v}$ and $\kappa_{v}$ can be simply identified from $G_{e, v}(s)$ and $P_{a, v}(s)$ with $G_{e, v}(s)=$ $1-e^{-\beta_{v^{s}}}$ and $P_{a, v}(s)=\frac{\kappa_{v}}{\beta_{v}}\left(1-e^{-\beta_{v^{s}}}\right)$. Since $\beta_{v}=\kappa_{v}+\sigma_{s, v}, \sigma_{s, v}$ is therefore easily obtained. Finally, $\phi_{v}\left(u, u^{\prime}\right)=\phi_{v}\left(\mu_{s}\right)$ is given by:

$$
\phi_{v}\left(\mu_{S}\right)=\frac{\int_{V_{F}} \int_{4 \pi} \int_{4 \pi} \delta\left(u \cdot u^{\prime}-\mu_{s}\right) \rho_{S i C, v} u \cdot n(u, x) d \Omega(u) d \Omega^{\prime}\left(u^{\prime}\right) d x}{\int_{V_{F}} \int_{4 \pi} \int_{4 \pi} \rho_{S i C, v} u . n(u, x) d \Omega(u) d \Omega^{\prime}\left(u^{\prime}\right) d x}
$$

with $\mu_{s}=u . u^{\prime}$ in reason of the statistical isotropy of the porous media. In Eq. (3) the sum over $V_{F}$ corresponds to random choices of a source points $M(x)$ within the fluid phase and $n(u, x)$ stands for the normal drawn from triangles of the mesh (obtained with genMat) localized at the position $x$ for the direction of incidence $u$.

\subsection{Vectorial finite elements method for modeling the radiative field at macroscale}

At macroscopic levels, in order to accurately model the field of radiation, discrete ordinates form of the radiative transfer equation (RTE) has been solved, see [22]. The discrete ordinates form RTE is a set of $N_{d}$ coupled partial differential equations. The $m$ th equation in this set represents the transport of the discrete radiative intensity $I_{m, v}(x)$ within the simulated domain and propagating along the direction $u_{m}$. The $m$ th equation in the set reads:

$$
\left(u_{m} . \nabla+\beta_{v}\right) I_{m, v}(x)-\kappa_{v} I_{b, v}-\sigma_{s, v} \sum_{n=1}^{N_{d}} \omega_{n} I_{n, v}(x) \phi_{v}\left(u_{m}, u_{n}\right)=0
$$

In this equation, $\beta_{v} I_{m, v}(x)$ is the radiation loss due to extinction, $\kappa_{v} I_{b, v}$ is the radiation gained due to internal emission, and $\sigma_{s, v} \sum_{n=1}^{n_{d}} \omega_{n} I_{n, v}(x) \phi_{v}\left(u_{m}, u_{n}\right)$ is the radiation gained due to in-scattering. The data $\beta_{v}, \kappa_{v}$, and $\sigma_{s, v}$ are obtained using previously explained approaches from sections 2.1 and 2.2. Finally, $\phi_{v}\left(u_{m}, u_{n}\right)$ is the anisotropic scattering probability phase function which we have modeled using the Henyey-Greenstein approximation and, $I_{b, v}$ is the Planckian black-body intensity within the homogenized medium.

To solve Eq. (4) in a robust manner, we have employed our previously introduced parallelized vectorial finite element based solver, see $[14,23]$. The main advantage of using the vectorial finite elements is that all $N_{d}$ radiative intensities can be approximated simultaneously to the same order of accuracy, and being based on finite elements method complex geometries, unstructured meshes, and complicated boundary conditions can be easily handled. Without attempting for completeness, we refer readers to Ref. [23, 24] where in particular the second-order accuracy of the method is extensively discussed for manufactured solutions [23]. In addition, the vectorial finite elements allows straightforward parallelization. Since parallel, high number of ordinates $\left(N_{d}=128\right)$ and spatial mesh resolution ( 0.1 million nodes) has been used to simulate radiative phenomena in foams at macroscopic levels. As such, the numerically constructed radiative intensity $I_{m, v}(x)$ can be said to be of high precision [23]. Further, we calculate the radiation propagation (radiative heat source) $Q_{\text {rad }}(x)$ using $I_{m, v}(x)$ :

$$
\nabla . Q_{\text {rad }}(x)=\int_{0}^{\infty} \kappa_{v}\left(4 \pi I_{b, v}-\sum_{m=1}^{N_{d}} \omega_{m} I_{m, v}(x)\right) d v
$$

Quantifying $Q_{\text {rad }}(x)$ within the homogenized samples of SiC foams, the samples character and performance for heat transfer applications can be judged. In the forthcoming results and discussion section we would show how chemical doping at microscopic level modifies $Q_{\text {rad }}(x)$ for different foam samples at macroscopic scales. 
IHTC16-24216

\section{RESULTS AND DISCUSSION}

\subsection{Microscopic evolution of the complex dielectric functions with the carrier concentration}

Simulations are carried out in the microcanonical ensemble on a ZB structure containing 13824, 8000 and 216 atoms which corresponds respectively to a volume of $12 \times 12 \times 12,10 \times 10 \times 10$, and $3 \times 3 \times 3$ cubic cells (the lattice constant is $4.3709 \AA$ ) with periodic boundary conditions, over a period of 100000 time steps of $0.8 f s$. This time is required to solve the second Newton's law by using the fourth order Gear predictor-corrector algorithm. The simulation box is equilibrated during 2000 time steps at the temperature of $300 \mathrm{~K}$ by using a velocity-scaling thermostat. As explained in the section 2.1, the doping is realized by substituting carbon atoms by nitrogen atoms through a random positioning process. Three concentrations are particularly highlighted in this work: $n_{c}=6.9$ $10^{18}, 1.210^{19}$, and $1.810^{21} \mathrm{~cm}^{-3}$, the intermediate concentration corresponding to the one previously studied by some of the authors in [10]. For each system, the complex dielectric function is computed according to the method described in section 2.1. As also explained, it allows the retrieval for each concentration of $v_{T O}, v_{L O P C}$, and $\Gamma$ in a first step, followed by $v_{P}$ and $\gamma_{P}$ in a second step.
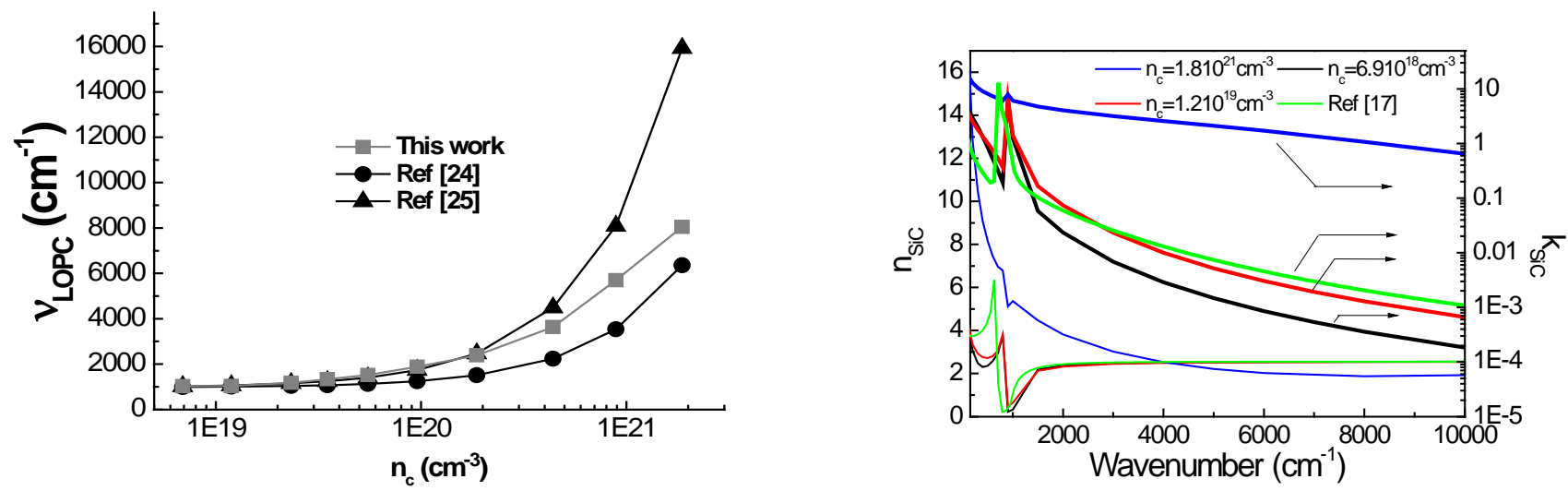

Fig.1 a) Carrier concentration dependence of $v_{L O P C}$ for virtual samples of doped $\mathrm{SiC}$ at $\mathrm{T}=300 \mathrm{~K} \mathrm{~b}$ ) Spectral dependence at $\mathrm{T}=300 \mathrm{~K}$ of the indices of refraction and of absorption of SiC crystalline domains with $n_{c}$

The Fig. 1a depicts the evolution of $v_{L O P C}$ for growing $n_{c}$. The evolution is in good agreement with previous analytical relations extracted from experimental works based on the characterization at $T=300 \mathrm{~K}$ with microRaman spectroscopy of the mode $v_{L O P C}$ of SiC layers with variable nitrogen doping [25, 26]. Since all the parameters for computing $\tilde{\epsilon}_{L D, v}$ for the three different carriers concentrations are now known, $\tilde{n}_{S i C, v}$ is easily obtained as it can be shown in the Fig. 1b. The thin and thick green curves give the spectral dependence at $T=300$ $\mathrm{K}$ of the complex refractive index of a doped $\mathrm{SiC}$ single crystal used to compute the radiative properties of opencell foams with growing porosities and nominal pore diameters [17]. The evolutions of the index of refraction $n_{S i C, v}$ and absorption $k_{S i C, v}$ match here completely those obtained by our molecular dynamic approach, knowing that for the two samples $n_{c}$ are rather identical: around $10^{19} \mathrm{~cm}^{-3}$ in [17] and $1.210^{19} \mathrm{~cm}^{-3}$ in this work (red curves). For the two lowest concentration, the values of $k_{S i C, v}$ are separated by a decade. For the highest concentration, the increase of $k_{S i C, v}$ is spectacular on the whole spectral range and $n_{S i C, v}$ is mainly changed in the far- and the midinfrared range that indicates a more pronounced reflective behavior.

\subsection{Mesoscopic temperature dependence of the homogenized radiative properties}

In this section, the complex refractive indices depicted in Fig. 1b will be assigned to describe the local optical response of SiC-based solid struts constituting open-cell foams.Then, 3 open-cell foams have been numerically generated using genMat (C++, Qt framework). Each foam has a cubic shape with an edge of $5 \mathrm{~mm}$ that corresponds 
IHTC16-24216

to a cube of 210 voxels width with a spatial resolution of $24 \mu \mathrm{m} /$ voxel. The targeted porosities are $40 \%, 70 \%$ and $90 \%$ for a nominal pore diameter around $1.9 \mathrm{~mm}$. Due to the numerical process of elaboration, well detailed furthermore in Ref. [12], pores have an ellipsoidal shape as it the case for real open-cell foam elaborated with the polymeric replication method $[1,6,7]$. We have verified that the 3 volumes are higher than the $R E V$ useful for the computation of the volumetric radiative properties. As explained in section 2.3, the collision-based Monte Carlo ray tracing is applied to compute for each foam $\beta_{v}, \kappa_{v}, \sigma_{s, v}$, and $\phi_{v}\left(\mu_{s}\right)$. Only the two values of $n_{C}$ which guarantees that struts are optically thick, are used for the subsequent calculations $\left(n_{C}=1.210^{19} \mathrm{~cm}^{-3}\right.$ and $n_{C}=1.8$ $10^{21} \mathrm{~cm}^{-3}$ ). Each simulation has been run with $10^{6}$ rays, the shooting area being a cube of 300 voxels centered on the numerical sample, for a computing time of $45 \mathrm{~s}$ on a desktop PC. Once $\beta_{v}, \kappa_{v}$, and $\sigma_{s, v}$ are obtained, their total quantities are determined by integrating their spectral contributions weighted by the spectral distribution defined by the Plank's law at a given temperature, $T$.
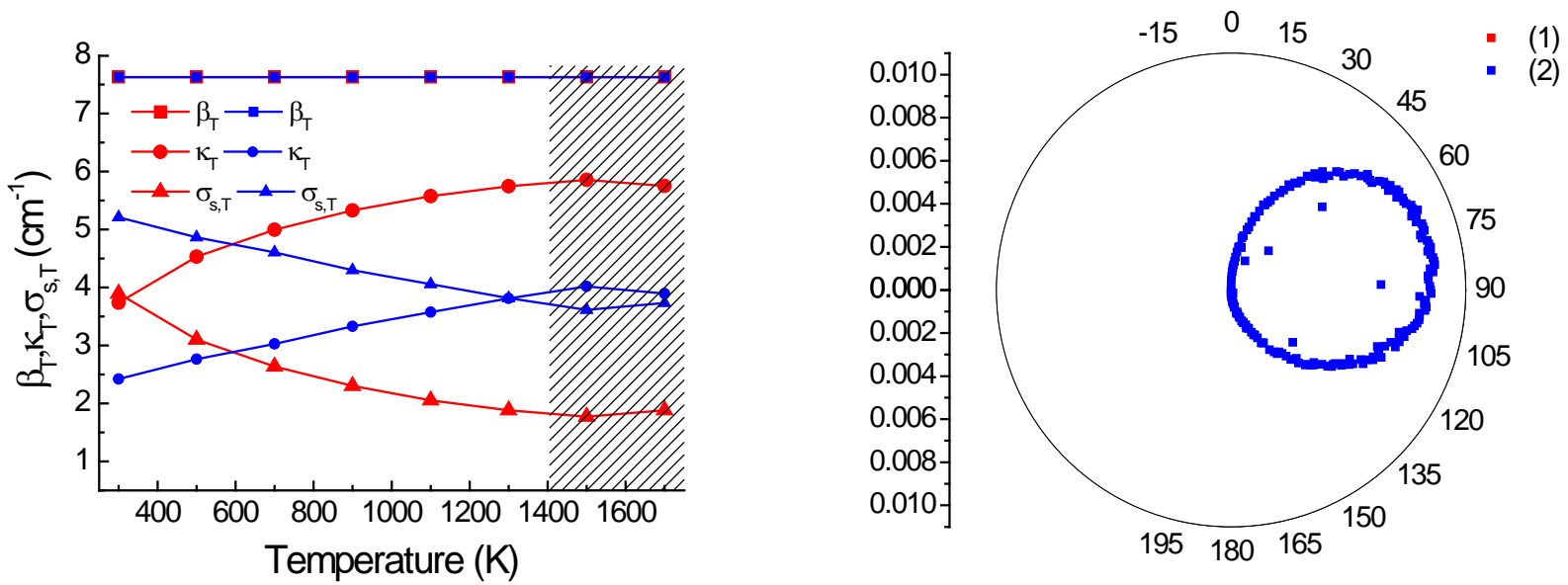

Fig.2 a) Temperature dependence of the volumetric radiative properties of two foams with $p=70 \%$ and $d_{\text {nom }}=$ $1.9 \mathrm{~mm}$ but $n_{c}=1.210^{19} \mathrm{~cm}^{-3}$ (red curves) and $n_{c}=1.810^{21} \mathrm{~cm}^{-3}$ (blue curves) b) Scattering phase functions at $T=$ $300 \mathrm{~K}$ of same foams with $n_{c}=1.210^{19} \mathrm{~cm}^{-3}$ (red curve) and $n_{c}=1.810^{21} \mathrm{~cm}^{-3}$ (blue curve)

The Fig. 2a details the evolution of $\beta_{T}, \kappa_{T}$, and $\sigma_{s, T}$ for the temperature range going from $300 \mathrm{~K}$ up to $1700 \mathrm{~K}$ for two foams with a porosity of $70 \%$, a nominal pore diameter of $1.9 \mathrm{~mm}$ but two carrier concentrations (red curves : $1.210^{19} \mathrm{~cm}^{-3}$, blue curves : $n_{C}=1.810^{21} \mathrm{~cm}^{-3}$ ). The evolutions are the same for the foams with $p=40 \%$ and 90 \%. In Fig. 2a, as expected, the temperature does not affect the values of $\beta_{T}$ since this quantity depends only on $p$ and on $d_{\text {nom }}$ also demonstrated in [12]. We consider $p$ and $d_{\text {nom }}$ remain constant with $T$. However, the changes are evident for $\kappa_{T}$ and $\sigma_{s, T}$. For $n_{c}=1.210^{19} \mathrm{~cm}^{-3}$ and $n_{c}=1.810^{21} \mathrm{~cm}^{-3}, \kappa_{T}$ increases with the temperature and $\sigma_{s, T}$ decreases in an opposite way. The more reflective behavior of the solid part with the highest carrier concentration explains why the associated foam has highest values for $\sigma_{s, T}$. In other words, the highest value of $n_{c}$ tends to decrease the absorption of an incident radiative flux. The striking modifications for $\kappa_{T}$ and $\sigma_{s, T}$ are not observed for $\phi_{v}\left(\mu_{s}\right)$ which exhibit a backscattering behavior whatever the doping is (see Fig. 2b).

\subsection{Macroscopic evolution of the radiative flux on a homogenized sample}

For the macroscopic numerical experiments, we simulated cubic geometry homogenized foam samples (edge of 5 $\mathrm{mm}$ ) with collimated radiative flux of $1000 \mathrm{~kW} / \mathrm{m}^{2}$ (solar concentration flux) impinging on the cubes left face (Dirichlet type boundary condition), but with different temperatures $(T=300$ and $1300 \mathrm{~K})$, different carrier concentrations $\left(n_{c}=1.210^{19}\right.$ and $\left.1.810^{21} \mathrm{~cm}^{-3}\right)$, and different porosities ( $p=40,70$, and $\left.90 \%\right)$. As mentionned in section 2.3 an advanced parallelized technique, vectorial finite element method, was employed to carry out the simulations using a fine angular discretization $N_{d}=128$ and an unstructured tetrahedral mesh of 0.1 million nodes. The computations were performed on an 8-core personal laptop within 2 minutes of runtime for each case. Data 
IHTC16-24216

for the homogenized radiative properties for these tests has been obtained from Fig. 3a. Note that HenyeyGreenstein scattering phase function constructed with anisotropic parameter $g=-0.6$ was used in our tests, since it provides a good estimate of the Monte-Carlo ray tracing constructed phase function in Fig. 2b. For these tests, the comparison of radiative sources plotted against the center line of the samples is presented in Fig. 3a and $3 \mathrm{~b}$.
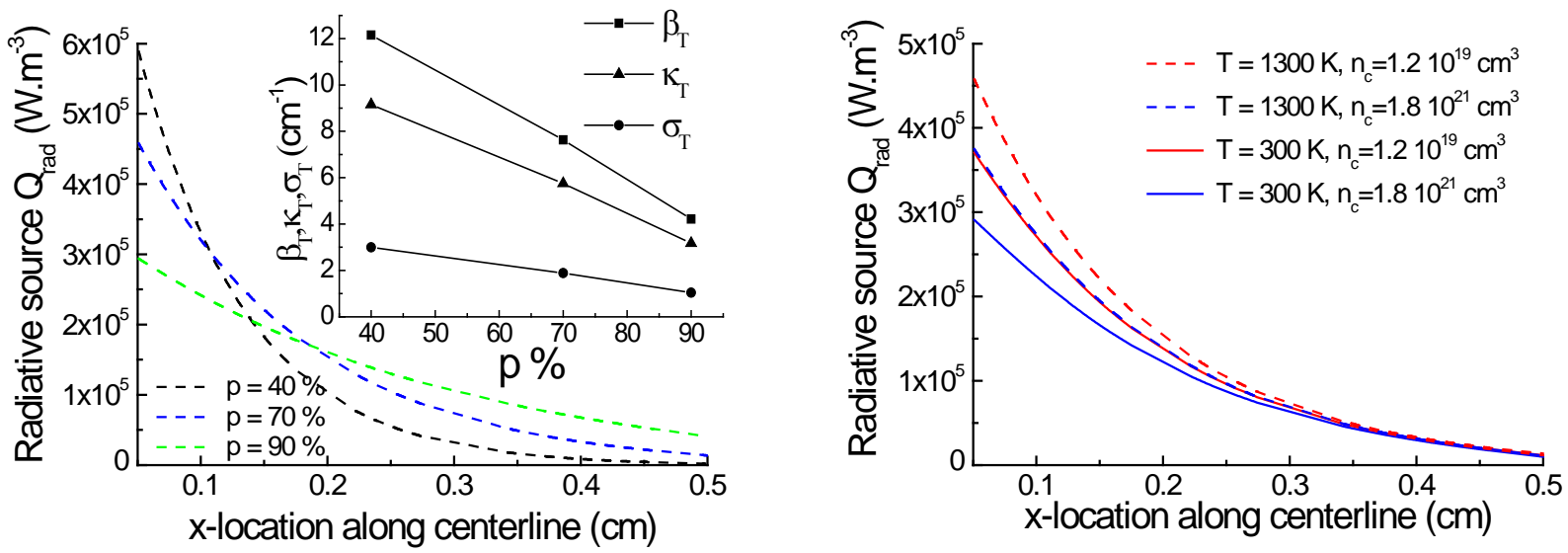

Fig 3 a) Propagation of incident radiation along the center line of $5 \mathrm{~mm}$ foam samples for $p=40,70,90 \%$ at $\mathrm{T}=1300 \mathrm{~K}$ and $n_{c}=1.210^{19} \mathrm{~cm}^{-3}$. In the inset, evolutions of the volumic radiative quantities with the porosity at $\mathrm{T}=1300 \mathrm{~K}$ and $n_{c}=1.210^{19} \mathrm{~cm}^{-3}$. b) Same propagation but for foam samples $p=70 \%, T=300$ (solid curves) and $1300 \mathrm{~K}$ (dashed curves) and $n_{c}=1.210^{19}$ (red color) and, $1.810^{21} \mathrm{~cm}^{-3}$ (blue color)

The Fig. 3a shows clearly that in the vicinity of the front face, the diminution of $\kappa_{T}$ for growing porosities leads to a decreasing of $Q_{\text {rad }}(x)$. Indeed, for a fixed value of $d_{\text {nom }}, \beta_{T}$ falls down when $p$ rises as was demonstrated in [12], as a consequence we obtain a lower value of $\kappa_{T}$. When the beam propagates within the foams, $Q_{\text {rad }}(x)$ decreases with a Beerian-like behavior as was also shown in [2, 27]. However, the lower the $p$ is, the more intense the diminution of $Q_{\text {rad }}(x)$ is. Conversely, for the highest value of $p$, the foam exhibits an optically thin behavior that do not allow to efficiently trap the incident radiation for solar-to-heat conversion. We observe that foams with $p=70 \%$ provide best compromise for efficiently depositing radiative energy within the inner volume. Fig. 3b, indicates what can be obtained with a heavily doped SiC-based foam. For chemically doped samples $\left(n_{c}=1.810^{21}\right.$ $\left.\mathrm{cm}^{-3}\right), Q_{\text {rad }}(x)$ drops $21.5 \%$ at $T=300 \mathrm{~K}$ and $18.1 \%$ at $T=1300 \mathrm{~K}$. When light penetrates within the foams, the effect of the chemical doping is more noticeable on $Q_{\text {rad }}(x)$ for the first rank of cells facing the incident beam, whereas $Q_{\text {rad }}(x)$ presents similar diminution when light arrives in the second mid part of the foams. A decrease of $Q_{\text {rad }}(x)$ near the front face, as it the case for $n_{c}=1.810^{21} \mathrm{~cm}^{-3}$, is likely to conduct to a diminution of $T$ as it is expected when a spectral selective material is used for designing volumetric solar receiver [2].

\section{CONCLUSION}

A combination of three numerical methods (molecular dynamics method, collision-based Monte Carlo ray tracing method, and vectorial finite element method) was used to finely tune, length scale by length scale, the radiative behavior of virtual SiC-based open cell foams up to $T=1300 \mathrm{~K}$. Practically, the homogenized radiative parameters of the foams are controlled here by three parameters: the porosity, the pore nominal diameter, and the carrier concentration. By controlling the chemical doping at the microscopic scale, the level of the complex dielectric function of the solid parts constituting the foams was altered. As such, modification of the absorbing and scattering behavior is evidenced for identical topology foams but growing chemical doping. In our macroscopic numerical experiments, we observed drop in radiative propagation in the vicinity of the front faces of heavily doped foams $\left(n_{c}=1.810^{21} \mathrm{~cm}^{-3}\right)$ in comparison with classical foams $\left(n_{c}=1.210^{19} \mathrm{~cm}^{-3}\right)$. This work can open new doors in the field of concentrated solar power engineering. 


\section{ACKNOWLEDGEMENT}

A.M.M and M.A.B. thank respectively the French Environment and Energy Management Agency and the French Institute in Research and Technology in Advance Manufacturing Technologies for Composite, Metallic and Hybrid Structures for funding their Ph-D thesis. The authors express also their gratitude to the members of the French GDR CNRS 3438 ACCORT for stimulating discussions.

\section{REFERENCE}

[1] Füssel A, Böttge D, Adler J, Marschallek F, Michaelis A. Cellular ceramics in combustion environments. Advanced Engineering Materials. 2011;13:1008-14.

[2] Kribus A, Gray Y, Grijnevich M, Mittelman G, Mey-Cloutier S, Caliot C. The promise and challenge of solar volumetric absorbers. Solar Energy. 2014;110:463-81.

[3] Aronovici M, Bianchi G, Ferrari L, Barbato M, Gianella S, Scocchi G, et al. Heat and Mass Transfer in Ceramic Lattices During HighTemperature Oxidation. Journal of the American Ceramic Society. 2015;98:2625-33.

[4] Saddow SE, Agarwal AK. Advances in silicon carbide processing and applications: Artech House; 2004.

[5] Suyama S, Kameda T, Itoh Y. Development of high-strength reaction-sintered silicon carbide. Diamond and Related Materials. 2003;12:1201-4.

[6] Tseng CC, Sikorski RL, Viskanta R, Chen MY. Effect of Foam Properties on Heat Transfer in High Temperature Open-Cell Foam Inserts. Journal of the American Ceramic Society. 2012;95:2015-21.

[7] Dietrich B, Fischedick T, Wallenstein M, Kind M. Thermal conductivity of ceramic sponges at temperatures up to 1000 C. Special Topics \& Reviews in Porous Media: An International Journal. 2015;6.

[8] Narita K, Hijikata Y, Yaguchi H, Yoshida S, Nakashima S. Characterization of carrier concentration and mobility in n-type SiC wafers using infrared reflectance spectroscopy. Japanese journal of applied physics. 2004;43:5151.

[9] Spitzer WG, Kleinman D, Walsh D. Infrared Properties of Hexagonal Silicon Carbide. Physical Review. 1959;113:127-32.

[10] Guévelou S, Rousseau B, Domingues G, Vicente J, Caliot C. Representative elementary volumes required to characterize the normal spectral emittance of silicon carbide foams used as volumetric solar absorbers. International Journal of Heat and Mass Transfer. 2016;93:118-29.

[11] Modest M. Radiative Heat Transfer: Academic Press; 2003.

[12] Guevelou S, Rousseau B, Domingues G, Vicente J, Flamant G, Caliot C. Evolution of the homogeneized volumetric radiative properties of a familliy of $\alpha$-SiC foams with growing nominal pore diameter Journal of Porous Media. 2015;18.

[13] Domingues G, Monthe AM, Guévelou S, Rousseau B. Study by molecular dynamics of the influence of temperature and pressure on the optical properties of undoped 3C-SiC structures. Journal of Quantitative Spectroscopy and Radiative Transfer. 2017.

[14] Badri M, Favennec Y, Jolivet P, Le Hardy D, Le Corre S, Rousseau B. Spatial versus angular parallelization of radiative transfer eqaution in participating media. ICHMT DIGITAL LIBRARY ONLINE: Begel House Inc.; 2017.

[15] Engelbrecht F, Helbig R. Effect of crystal anisotropy on the infrared reflectivity of 6H-SiC. Physical Review B. 1993;48:15698.

[16] Ferro G, Chaussende D. A new model for in situ nitrogen incorporation into 4H-SiC during epitaxy. Scientific Reports. $2017 ; 7$.

[17] Rousseau B, Guevelou S, Mekeze-Monthe A, Vicente J, Del Campo L, Meneses DDS, et al. Tuning the spectral emittance of $\alpha$-SiC open-cell foams up to $1300 \mathrm{~K}$ with their macro porosity. AIP Advances. 2016;6:065226.

[18] Tersoff J. Modeling solid-state chemistry: Interatomic potentials for multicomponent systems. Physical Review B. 1989;39:5566.

[19] Halac E, Burgos E, Bonadeo H. Static and dynamical properties of SiC polytypes. Physical Review B. 2002;65:125202.

[20] Ridley B. On the effect of plasmon-phonon coupling and phonon reabsorption on scattering-induced NDR. Superlattices and Microstructures. 1986;2:159-64.

[21] Guévelou S, Rousseau B, Domingues G, Vicente J. A simple expression for the normal spectral emittance of open-cell foams composed of optically thick and smooth struts. Journal of Quantitative Spectroscopy and Radiative Transfer. 2017;189:329-38.

[22] Fiveland W, Jessee J. Finite element formulation of the discrete-ordinates method for multidimensional geometries. Journal of thermophysics and heat transfer. 1994;8:426-33.

[23] Badri MA, Jolivet P, Rousseau B, Le Corre S, Digonnet H, Favennec Y. Vectorial finite elements for solving the radiative transfer equation. Journal of Quantitative Spectroscopy and Radiative Transfer. 2018;212:59-74.

[24] Boffi D, Brezzi F, Fortin M. Mixed finite element methods and applications: Springer; 2013.

[25] Nakashima S, Kitamura T, Kato T, Kojima K, Kosugi R, Okumura H, et al. Determination of free carrier density in the low doping regime of 4 H-Si C by Raman scattering. Applied Physics Letters. 2008;93:121913.

[26] Peng Y, Xu X, Hu X, Jiang K, Song S, Gao Y, et al. Raman spectroscopic study of the electrical properties of 6H-SiC crystals grown by hydrogen-assisted physical vapor transport method. Journal of Applied Physics. 2010;107:093519.

[27] Gomez-Garcia F, González-Aguilar J, Olalde G, Romero M. Thermal and hydrodynamic behavior of ceramic volumetric absorbers for central receiver solar power plants: A review. Renewable and Sustainable Energy Reviews. 2016;57:648-58. 